\title{
Two-Loop Helicity Amplitudes for Diphoton Plus Jet Production in Full Color
}

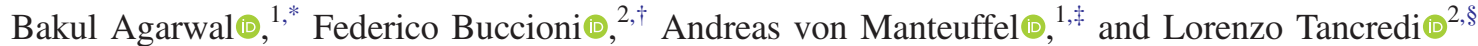 \\ ${ }^{1}$ Department of Physics and Astronomy, Michigan State University, East Lansing, Michigan 48824, USA \\ ${ }^{2}$ Rudolf Peierls Centre for Theoretical Physics, University of Oxford, \\ Clarendon Laboratory, Parks Road, Oxford OXI 3PU, United Kingdom
}

(Received 15 May 2021; revised 27 September 2021; accepted 30 November 2021; published 23 December 2021)

\begin{abstract}
We present the two-loop QCD amplitudes for the production of two photons and a jet at hadron colliders with full-color dependence. This is the first time that radiative corrections for a five-particle scattering process have been computed beyond the leading-color approximation at this perturbative order in QCD. The results presented in this Letter will be crucial to guaranteeing reliable predictions with unprecedented precision for diphoton production at hadron colliders. The methodologies that we describe lead to a significant simplification of the calculation and their applicability extends to a wider range of five-point scattering processes.
\end{abstract}

DOI: 10.1103/PhysRevLett.127.262001

The past two decades have witnessed impressive developments in our understanding of scattering amplitudes in quantum field theory, both in supersymmetric theories such as $\mathcal{N}=4$ super Yang-Mills, and in phenomenologically relevant ones such as quantum chromodynamics (QCD). The increasing interest devoted to formal aspects of scattering amplitudes is motivated by their fundamental role in providing accurate theoretical predictions to interpret the experimental data collected at particle colliders like the CERN Large Hadron Collider (LHC). The LHC experiments are very successful at measuring a multitude of interesting physical observables with unprecedented precision, opening the way to a new era of precision collider physics. Comparing high-quality data with accurate theoretical predictions for wisely constructed physical observables offers a promising opportunity to unveil so-far elusive signs of new physics beyond the standard model. The required theoretical calculations can be performed in perturbative quantum field theory by means of series expansions in the relevant coupling constants, which are assumed to be small. The coefficients of the perturbative series are expressed in terms of Feynman diagrams of rising complexity, i.e., with an increasing number of external legs and internal loops.

While a general mathematical understanding of scattering amplitudes in quantum field theory remains beyond reach, important breakthroughs have been achieved in the past years. In particular, the interplay of techniques

Published by the American Physical Society under the terms of the Creative Commons Attribution 4.0 International license. Further distribution of this work must maintain attribution to the author(s) and the published article's title, journal citation, and DOI. Funded by SCOAP ${ }^{3}$. borrowed from particle physics phenomenology [1-7] and $\mathcal{N}=4$ super Yang-Mills [8-11] with developments in the theory of special functions [12-21] resulted in a toolkit that allowed the community to compute a large set of physically interesting $2 \rightarrow 2$ processes in two-loop QCD. The steadily improving performance of the LHC experiments has contributed to pushing the theory calculations further. The first three-loop corrections to $2 \rightarrow 2$ scattering amplitudes in supersymmetric theories [22-24] and, very recently, in full QCD [25] have been computed. Concurrently, considerable effort has been put in understanding the properties of higher-multiplicity processes, most prominently $2 \rightarrow 3$ scattering at two loops. This program profited from breakthroughs both in the development of new strategies for handling their extraordinary algebraic complexity [26-35], and in the study of the special functions required for their evaluation [36-39]. These advances allowed the calculation of two-loop leading-color corrections to various $2 \rightarrow 3$ processes [40-56], which have made it possible to perform the first phenomenological studies in next-to-next-to-leading-order (NNLO) leading-color QCD for three-photon production at the LHC [57,58]. While the leading-color approximation often provides a realistic estimate of the bulk of the corrections, its validity is not guaranteed a priori for arbitrary processes at the target precision. This motivates a full-color calculation of the two-loop amplitudes to enable reliable phenomenological predictions. Subleadingcolor corrections are also interesting for formal reasons, as they typically require the computation of complicated nonplanar Feynman diagrams and are therefore the first place where the full complexity of two-loop scattering amplitudes becomes manifest. Although all nonplanar master integrals for $2 \rightarrow 3$ massless processes are now available [39], their use in the actual calculation of physical 
amplitudes poses additional challenges and has remained, as of today, an outstanding task; full results in QCD have been obtained only for five-gluon scattering in the substantially simpler all-equal helicity configuration [59].

In this Letter, we fill the gap and compute, for the first time, the full-color two-loop massless QCD corrections for a genuine $2 \rightarrow 3$ scattering process at the LHC, the production of two photons and a strongly interacting jet. This process is of foremost phenomenological interest: comparing measurements of pairs of photons with nonzero transverse momentum at the LHC with accurate theory predictions allows us put stringent constraints on various beyond-standard-model scenarios. At the technical level, the calculation provides a nontrivial benchmark involving colored particles in the initial and final states. Finally, these amplitudes constitute an essential ingredient for the $\mathrm{N}^{3} \mathrm{LO} \mathrm{QCD}$ corrections to diphoton production at the LHC.

The production of two photons and a parton in protonproton collisions proceeds through three classes of partonic channels, namely quark-antiquark annihilation, $q \bar{q} \rightarrow g \gamma \gamma$, quark(antiquark)-gluon scattering, $q(\bar{q}) g \rightarrow q(\bar{q}) \gamma \gamma$, and the loop-induced gluon-fusion channel, $g g \rightarrow g \gamma \gamma$. The two-loop corrections to the latter formally contribute starting at $\mathrm{N}^{3} \mathrm{LO}$ and we do not consider them in this Letter. We focus instead on the channels that involve a pair of quarks and define the three subprocesses as

$$
\begin{aligned}
& q\left(p_{1}\right)+\bar{q}\left(p_{2}\right) \rightarrow g\left(p_{3}\right)+\gamma\left(p_{4}\right)+\gamma\left(p_{5}\right), \\
& q\left(p_{1}\right)+g\left(p_{2}\right) \rightarrow q\left(p_{3}\right)+\gamma\left(p_{4}\right)+\gamma\left(p_{5}\right), \\
& g\left(p_{1}\right)+\bar{q}\left(p_{2}\right) \rightarrow \bar{q}\left(p_{3}\right)+\gamma\left(p_{4}\right)+\gamma\left(p_{5}\right) .
\end{aligned}
$$

All external particles are massless, $p_{i}^{2}=0$ for $i=1, \ldots, 5$, thus the kinematics is completely fixed in terms of five independent kinematic invariants, which we choose as

$s_{12}=\left(p_{1}+p_{2}\right)^{2}, \quad s_{23}=\left(p_{2}-p_{3}\right)^{2}, \quad s_{34}=\left(p_{3}+p_{4}\right)^{2}$,

$s_{45}=\left(p_{4}+p_{5}\right)^{2}, \quad s_{15}=\left(p_{1}-p_{5}\right)^{2}$.

The physical scattering region is identified by [37]

$$
\begin{aligned}
s_{12} & \geq s_{34}>0, \quad s_{12}-s_{34} \geq s_{45}>0, \\
0 & \geq s_{23} \geq s_{45}-s_{12}, \quad s_{15}^{-} \leq s_{15} \leq s_{15}^{+}, \quad s_{15}<0,
\end{aligned}
$$

where

$$
\begin{aligned}
s_{15}^{ \pm}= & \frac{1}{\left(s_{12}-s_{45}\right)^{2}}\left[s_{12}^{2} s_{23}+s_{34} s_{45}\left(s_{45}-s_{23}\right)\right. \\
& -s_{12}\left(s_{34} s_{45}+s_{23} s_{34}+s_{23} s_{45}\right) \\
& \left. \pm \sqrt{s_{12} s_{23} s_{34} s_{45}\left(s_{12}+s_{23}-s_{45}\right)\left(s_{34}+s_{45}-s_{12}\right)}\right]
\end{aligned}
$$

In order to describe these five-point amplitudes, it is also useful to introduce the parity-odd invariant

$$
\epsilon_{5}=4 i \epsilon_{\mu \nu \rho \sigma} p_{1}^{\mu} p_{2}^{\nu} p_{3}^{\rho} p_{4}^{\sigma},
$$

where $\epsilon_{\mu \nu \rho \sigma}$ is the totally antisymmetric Levi-Civita symbol $\left(\epsilon_{5}\right)^{2}=\Delta$, and

$$
\begin{aligned}
\Delta= & -4 s_{12} s_{23} s_{34}\left(s_{23}-s_{45}-s_{15}\right) \\
& +\left(s_{12} s_{23}+s_{23} s_{34}-s_{34} s_{45}+s_{45} s_{15}-s_{15} s_{12}\right)^{2},
\end{aligned}
$$

is the determinant of the Gram matrix $\left(G_{i j}\right)=\left(2 p_{i} p_{j}\right)$. In the scattering physical region, $\Delta<0$ and $\epsilon_{5}= \pm i \sqrt{|\Delta|}$, where the sign depends on the actual kinematics inside this region [60].

We first focus on the quark-antiquark annihilation $(q \bar{q})$ channel in (1), and describe details specific to the crossed channels (2), (3) afterward. We express the amplitude as

$$
A_{i j}^{a}=i(4 \pi \alpha) Q_{q}^{2} \sqrt{4 \pi \alpha_{s}} \mathbf{T}_{i j}^{a} \mathcal{A} .
$$

Here, $\alpha$ is the fine structure constant, $\alpha_{s}$ is the strong coupling constant, $Q_{q}$ is the quark charge in units of the electron charge, $i, j$ are the color indices of the $q \bar{q}$ pair, $a$ is the gluon color index, and $\mathbf{T}_{i j}^{a}$ are the $\mathrm{SU}(3)$ color generators in the fundamental representation.

We extract the helicity amplitudes for this process following the approach suggested in $[61,62]$. Specifically, we work in conventional dimensional regularization, but construct projectors to extract only the physically relevant helicity amplitudes. This is achieved by decomposing the amplitude into Lorentz structures which are independent in four dimensions, thereby avoiding the introduction of evanescent form factors. We write

$$
\mathcal{A}=\mathcal{A}^{\mu \nu \rho} \epsilon_{\mu}^{*}\left(p_{3}\right) \epsilon_{\nu}^{*}\left(p_{4}\right) \epsilon_{\rho}^{*}\left(p_{5}\right),
$$

where $\epsilon_{\mu}^{*}\left(p_{3}\right)$ is the polarization vector of the gluon, and $\epsilon^{*}\left(p_{j}\right)$ with $j=4,5$ are the polarization vectors of the photons. Imposing transversality, $\epsilon_{i} \cdot p_{i}=0$ for $i=3,4,5$, and making the cyclic gauge choice $\epsilon_{3} \cdot p_{4}=\epsilon_{4} \cdot p_{5}=\epsilon_{5} \cdot p_{1}=0$, we obtain

$$
\mathcal{A}=\sum_{j=1}^{16} F_{j} \mathcal{T}_{j},
$$

with the 16 Lorentz structures

$$
\begin{aligned}
\mathcal{T}_{1 \leq j \leq 8} & =\bar{u}\left(p_{2}\right) \not{ }_{3} u\left(p_{1}\right) t_{j}^{\mu \nu \rho} \epsilon_{\mu}^{*}\left(p_{3}\right) \epsilon_{\nu}^{*}\left(p_{4}\right) \epsilon_{\rho}^{*}\left(p_{5}\right), \\
\mathcal{T}_{9 \leq j \leq 16} & =\bar{u}\left(p_{2}\right) \not \not 1_{4} u\left(p_{1}\right) t_{j-8}^{\mu \nu \rho} \epsilon_{\mu}^{*}\left(p_{3}\right) \epsilon_{\nu}^{*}\left(p_{4}\right) \epsilon_{\rho}^{*}\left(p_{5}\right),
\end{aligned}
$$

where the tensors $t_{j}^{\mu \nu \rho}$ are given by 


$$
\begin{array}{ll}
t_{1}^{\mu \nu \rho}=p_{1}^{\mu} p_{1}^{\nu} p_{2}^{\rho}, & t_{2}^{\mu \nu \rho}=p_{1}^{\mu} p_{1}^{\nu} p_{3}^{\rho}, \\
t_{3}^{\mu \nu \rho}=p_{1}^{\mu} p_{2}^{\nu} p_{2}^{\rho}, & t_{4}^{\mu \nu \rho}=p_{1}^{\mu} p_{2}^{\nu} p_{3}^{\rho}, \\
t_{5}^{\mu \nu \rho}=p_{2}^{\mu} p_{1}^{\nu} p_{2}^{\rho}, & t_{6}^{\mu \nu \rho}=p_{2}^{\mu} p_{1}^{\nu} p_{3}^{\rho}, \\
t_{7}^{\mu \nu \rho}=p_{2}^{\mu} p_{2}^{\nu} p_{2}^{\rho}, & t_{8}^{\mu \nu \rho}=p_{2}^{\mu} p_{2}^{\nu} p_{3}^{\rho} .
\end{array}
$$

We stress that the number of independent Lorentz structures matches the number of helicity configurations.

Each form factor $F_{j}$ in Eq. (10) can then be computed by defining a projector

$$
\mathcal{P}_{j}=\sum_{k=1}^{16} c_{k}^{(j)} \mathcal{T}_{k}^{\dagger}
$$

with $c_{k}^{j}$ being rational functions of the Mandelstam invariants such that

$$
F_{j}=\sum_{\text {pol }} \mathcal{P}_{j} \mathcal{A},
$$

where the polarization sums read

$$
\begin{gathered}
\sum_{\mathrm{pol}} u(p) \bar{u}(p)=\not p, \\
\sum_{\mathrm{pol}} \epsilon_{\mu}(p) \epsilon_{\nu}^{*}(p)=-g_{\mu \nu}+\frac{p_{\mu} q_{\nu}+p_{\nu} q_{\mu}}{p \cdot q},
\end{gathered}
$$

with $q=p_{4}, p_{5}, p_{1}$ for $p=p_{3}, p_{4}, p_{5}$, respectively. We stress that even if, by construction, the algebra to derive the projectors is performed in $d$ space-time dimensions, the coefficients $c_{k}^{(j)}$ in Eq. (13) do not depend on $d$; see [61,62].

It is straightforward to compute helicity amplitudes from Eq. (10) by evaluating the Lorentz structures of Eq. (11) for specific helicities of the external particles in four dimensions. We denote the dependence of the color-stripped amplitude (9) on the external helicities by

$$
\mathcal{A}(\lambda) \text { with } \lambda=\left\{\lambda_{q}, \lambda_{3}, \lambda_{4}, \lambda_{5}\right\},
$$

where $\lambda_{q}=L, R$ is the helicity of the quark line, and $\lambda_{j}= \pm$ for $j=3,4,5$ are the helicities of the gluon and the two photons, respectively. For each partonic subchannel there are three independent helicity amplitudes, from which all the remaining ones can be obtained using parity, charge conjugation, and permutations of the external photons. We choose the following independent configurations

$$
\begin{aligned}
& \lambda_{A}=\{L,+,+,+\}, \\
& \lambda_{B}=\{L,-,+,+\}, \\
& \lambda_{C}=\{L,-,-,+\} .
\end{aligned}
$$

Note that $\mathcal{A}\left(\lambda_{A}\right)$ is zero at tree level. For each helicity amplitude we factor out either the tree amplitude if it is nonzero or a suitable product of spinors carrying the spinor weight of the amplitude. Employing the spinor-helicity formalism we write for left-handed spinors $\bar{u}_{L}\left(p_{2}\right)=\langle 2|$ and $\left.u_{L}\left(p_{1}\right)=\mid 1\right]$ and for a gauge boson of momentum $p_{j}$

$$
\epsilon_{j,-}^{\mu}\left(p_{j}\right)=\frac{\left\langle q_{j}\left|\gamma^{\mu}\right| j\right]}{\sqrt{2}\left\langle q_{j} j\right\rangle}, \quad \epsilon_{j,+}^{\mu}\left(p_{j}\right)=\frac{\left\langle j\left|\gamma^{\mu}\right| q_{j}\right]}{\sqrt{2}\left[j q_{j}\right]},
$$

where $q_{j}$ is the gauge fixing momentum. We then define spinor-free amplitudes $\mathcal{B}(\lambda)$ according to

$$
\mathcal{A}(\lambda)=\Phi(\lambda) \mathcal{B}(\lambda),
$$

where for the spinor functions $\Phi(\lambda)$ we choose $[55,63]$

$$
\begin{aligned}
& \Phi\left(\lambda_{A}\right)=2 \sqrt{2} \frac{[31]\langle 12\rangle^{3}\langle 13\rangle}{\langle 14\rangle^{2}\langle 15\rangle^{2}\langle 23\rangle^{2}}, \\
& \Phi\left(\lambda_{B}\right)=2 \sqrt{2} \frac{\langle 12\rangle\langle 23\rangle^{2}}{\langle 14\rangle\langle 42\rangle\langle 25\rangle\langle 51\rangle}, \\
& \Phi\left(\lambda_{C}\right)=2 \sqrt{2} \frac{[51]^{2}[12]}{[14][42][23][31]} .
\end{aligned}
$$

The corresponding phases for the $q g$ and $g \bar{q}$ channels can be obtained by exchanging $2 \leftrightarrow 3$ and $1 \leftrightarrow 3$, respectively.

The spinor-free amplitudes $\mathcal{B}(\lambda)$ can be decomposed into parity even and odd contributions,

$$
\mathcal{B}(\lambda)=\mathcal{B}^{E}(\lambda)+\bar{\epsilon}_{5} \mathcal{B}^{O}(\lambda),
$$

where $\bar{\epsilon}_{5}=\epsilon_{5} / s_{12}^{2}$ is dimensionless. We stress here that $\bar{\epsilon}_{5}$ changes sign under parity transformations or odd permutations of the external momenta; see Eq. (6). The $\mathcal{B}^{P}(\lambda)$ terms, with $P=E, O$, can in turn be expressed as linear combinations of the form factors in Eq. (10) with coefficients that depend only on the $s_{i j}$ [61,62]. From the operators in Eq. (13) one can finally derive six helicity amplitude projectors, decomposed in terms of the complex conjugates of the tensors in Eq. (11), and which directly project onto the $\mathcal{B}^{P}(\lambda)$ defined above. The explicit form of these projectors can be obtained from the authors upon request.

The QCD corrections can be computed by expanding the amplitudes in the bare strong coupling constant $\alpha_{s}^{b}$,

$$
\mathcal{B}^{P}(\lambda)=\sum_{k=0}^{2}\left(\frac{\alpha_{s}^{b}}{2 \pi}\right)^{k} \mathcal{B}^{P,(k)}(\lambda)+\mathcal{O}\left(\left(\alpha_{s}^{b}\right)^{3}\right) .
$$

By construction, the first order reads

$$
\begin{array}{ll}
\mathcal{B}^{E,(0)}\left(\lambda_{A}\right)=0, & \mathcal{B}^{O,(0)}\left(\lambda_{A}\right)=0, \\
\mathcal{B}^{E,(0)}\left(\lambda_{B}\right)=1, & \mathcal{B}^{O,(0)}\left(\lambda_{B}\right)=0, \\
\mathcal{B}^{E,(0)}\left(\lambda_{C}\right)=1, & \mathcal{B}^{O,(0)}\left(\lambda_{C}\right)=0 .
\end{array}
$$


We calculate the one- and two-loop corrections $\mathcal{B}^{P,(k)}$, $k=1,2$, as follows. First, we generate the Feynman diagrams using Qgraf [64]. We then contract them with the helicity amplitude projectors described above and express the amplitudes in terms of scalar Feynman integrals. All the algebra required at this level has been carried out using FORM [65]. The two-loop corrections can be mapped to a large number of scalar two-loop integrals drawn from two different integral families, which we describe in Supplemental Material [66].

As it is well known, the scalar integrals fulfill linear relations and can be reduced to a set of master integrals using symmetry relations and integration-by-parts identities (IBPs) [1,2]. Laporta's algorithm [67] maps the problem to the row reduction of a large matrix. While straightforward in principle, this step can become computationally very challenging and, until now, has remained a major bottleneck for the calculation of the nonplanar twoloop corrections to $2 \rightarrow 3$ massless scattering amplitudes. (Very recently, the reduction of the most complicated nonplanar rank five integrals has been achieved independently in [68].) We succeed in reducing all planar and nonplanar two-loop Feynman integrals as follows. First, we use REDUZE2 [69] to identify shift, symmetry, and crossing relations of the scalar integrals. The actual integration-byparts reduction of the remaining integrals is performed using a private implementation of the Laporta algorithm FINRED, augmented by the use of finite-field arithmetics $[32,33,70]$, syzygy techniques [27,71-74] and denominator guessing [44,75]. Despite expressing the integrals directly in terms of the canonical basis defined in [39], the reduction identities for the nonplanar integrals are quite cumbersome if the rational coefficients are represented in a common-denominator form, for example, with FERMAT [76]. As it has already been observed in previous work $[51,53,75,77,78]$, substantial simplifications of these identities can be achieved by a multivariate partial fraction decomposition of the rational coefficients. (The most complicated IBPs in our calculation are for rank-five nonplanar integrals, with sizes of order 1 GB each. Multivariate partial fractioning reduces their size by factors of order 100.) In practice, we find it most efficient to first perform the reduction for a minimal subset of integrals in the uncrossed families $\{A, B\}$, simplify them using partial fractioning, and finally cross them. For the partial fraction decomposition we employ MULTIVARIATEAPART [75], where we use SINGULAR [79] as a back end for the polynomial reductions. In this way, we produce all required identities for the reduction of the amplitude in a compact representation. After inserting the reduction identities in the amplitude we perform another partial fraction decomposition and express our results in terms of the pentagon functions defined in [39].

The bare amplitudes contain poles in the dimensional regulator $\epsilon$ both of ultraviolet (UV) and infrared (IR) origin.
We remove the UV poles by expressing our result in terms of the $\overline{\mathrm{MS}}$ renormalized strong coupling $\alpha_{s}(\mu)$ :

$$
\mathcal{B}^{P}(\lambda)=\sum_{k=0}^{2}\left(\frac{\alpha_{s}(\mu)}{2 \pi}\right)^{k} \overline{\mathcal{B}}^{P,(k)}(\lambda)+\mathcal{O}\left(\alpha_{s}^{3}\right),
$$

where the relation between renormalized and bare coupling is given by

$$
S_{\epsilon} \alpha_{s}=\mu^{2 \epsilon} \alpha_{s}(\mu) Z\left[\alpha_{s}(\mu)\right],
$$

with $S_{\epsilon}=\left(4 \pi e^{\gamma_{E}}\right)^{-\epsilon}$. The UV-renormalized amplitudes $\overline{\mathcal{B}}^{P,(k)}(\lambda)$ still contain divergences of IR origin. Following [80], their structure can be universally expressed in terms of the lower-order scattering amplitudes as

$$
\begin{aligned}
& \overline{\mathcal{B}}^{P,(1)}(\lambda)=\mathcal{I}_{1} \overline{\mathcal{B}}^{P,(0)}(\boldsymbol{\lambda})+\mathcal{R}^{P,(1)}(\lambda), \\
& \overline{\mathcal{B}}^{P,(2)}(\lambda)=\mathcal{I}_{2} \overline{\mathcal{B}}^{P,(0)}(\lambda)+\mathcal{I}_{1} \overline{\mathcal{B}}^{P,(1)}(\lambda)+\mathcal{R}^{P,(2)}(\lambda),
\end{aligned}
$$

where the $\mathcal{I}_{k}$ are universal and depend only on the loop order and on the species of the colored partons in the initial and final state of the scattering process, and the $\mathcal{R}^{P,(k)}(\lambda)$ are the so-called finite remainders. The explicit expressions of the operators $Z\left[\alpha_{s}\right]$ and $\mathcal{I}_{k}$ with $k=1,2$ are provided in the Supplemental Material [66].

It is convenient to organize the one- and two-loop corrections to the finite remainders $\mathcal{R}^{P,(k)}(\lambda)$ into separately gauge-invariant color structures

$$
\begin{aligned}
& \mathcal{R}^{P,(1)}(\lambda)=\sum_{i=1}^{4} \tilde{b}_{i} \mathcal{R}_{i}^{P,(1)}(\lambda), \\
& \mathcal{R}^{P,(2)}(\lambda)=\sum_{i=1}^{10} \tilde{c}_{i} \mathcal{R}_{i}^{P,(2)}(\lambda) .
\end{aligned}
$$

At one-loop we choose the following basis,

$$
\tilde{b}_{1}=N, \quad \tilde{b}_{2}=N^{-1}, \quad \tilde{b}_{3}=n_{f}^{\gamma \gamma}, \quad \tilde{b}_{4}=n_{f},
$$

whereas at two loop we have

$$
\begin{aligned}
\tilde{c}_{1} & =N^{2}, \quad \tilde{c}_{2}=1, \quad \tilde{c}_{3}=N^{-2}, \\
\tilde{c}_{4} & =N n_{f}, \quad \tilde{c}_{5}=N^{-1} n_{f}, \quad \tilde{c}_{6}=n_{f}^{\gamma \gamma} n_{f}, \\
\tilde{c}_{7} & =N n_{f}^{\gamma \gamma}, \quad \tilde{c}_{8}=N^{-1} n_{f}^{\gamma \gamma}, \quad \tilde{c}_{9}=n_{f}^{\gamma}\left(N-4 N^{-1}\right), \\
\tilde{c}_{10} & =n_{f}^{2},
\end{aligned}
$$

where $N$ is the number of colors, $n_{f}$ is the number of massless quarks, and $n_{f}^{\gamma}$ and $n_{f}^{\gamma \gamma}$ are defined as

$$
n_{f}^{\gamma}=\frac{1}{Q_{q}} \sum_{i=1}^{n_{f}} Q_{i}, \quad n_{f}^{\gamma \gamma}=\frac{1}{Q_{q}^{2}} \sum_{i=1}^{n_{f}} Q_{i}^{2} .
$$


The finite remainders $\mathcal{R}_{i}^{P,(k)}(\lambda)$ are formally expressed as linear combinations of the pentagon functions defined in [39], whose coefficients are rational functions of the kinematic invariants $s_{i j}$. After finding a minimal set of such rational functions, we perform an optimized partial fraction decomposition which removes spurious denominators and unnecessarily high denominator powers throughout. This two-step procedure, detailed in [53], renders our final expressions very compact and suitable for fast and stable numerical evaluations. Notably, for the most complicated color factor which involves nonplanar contributions, $c_{2}$ in Eq. (30), the largest helicity coefficient amounts to only $4.5 \mathrm{MB}$ in size.

The discussion presented so far focused on the $q \bar{q}$ partonic channel in Eq. (1), but the formal expressions of Eq. (28) hold for the crossed channels in Eqs. (2) and (3) as well. In fact, results for these processes can be readily extracted from the $q \bar{q}$ case without repeating any of the heavy steps of the calculation. In practice, we obtain the helicity amplitudes for the $g \bar{q}$ and $q g$ channels from the $q \bar{q}$ ones by applying the permutations $1 \leftrightarrow 3$ and $2 \leftrightarrow 3$, respectively. While this operation is trivial for rational functions of the $s_{i j}$ and $\bar{\epsilon}_{5}$, this is not the case for the pentagon functions. In order to express the crossed pentagon functions in terms of the uncrossed ones, we proceed as follows. First, we express the two-loop master integrals and crossings thereof in terms of pentagon functions. Second, we exploit the fact that the full set of master integrals is linearly mapped onto itself by any crossing of the external legs. By combining and reducing these systems of equations, we obtain reduction identities for the pentagon functions with permutations $1 \leftrightarrow 3$ and $2 \leftrightarrow 3$. These solutions provide us with sufficient information to successfully cross the helicity amplitudes and express them in terms of the original pentagon functions.

We perform various checks on our results. First, we verify that our tree-level and one-loop helicity amplitudes agree with the OPENLOOPS2 program [81]. Next, we check that the poles of our two-loop amplitudes follow the pattern predicted by Catani, and we compare the leadingcolor part of our two-loop helicity amplitudes with the results of [56] and find agreement. Finally, we perform a separate calculation of the interference of the two-loop and tree-level amplitudes summed over polarizations, both for the $q \bar{q}$ and for the $q g$ channels. In this second calculation no projectors are used, and the $q g$ channel is obtained by crossing the relevant amplitudes prior to reduction to master integrals instead of crossing the pentagon functions. After subtracting the UV and IR poles, we find perfect agreement for the four-dimensional finite remainder of all color factors between the two calculations, which provides a very strong check of our helicity amplitudes.
TABLE I. One- and two-loop finite remainders for diphoton plus parton production processes. Samples are shown for all independent helicity configurations $\lambda(18)$ in the kinematic point (35), including either full color, $\mathcal{R}^{(l)}(\lambda), l=1,2$, (32), or only two-loop leading-color contributions, $\mathcal{R}_{\mathrm{LC}}^{(2)}(\lambda)$ (33).

\begin{tabular}{ccc}
\hline \hline & $u \bar{u} \rightarrow g \gamma \gamma$ & $u g \rightarrow u \gamma \gamma$ \\
\hline $\mathcal{R}^{(1)}\left(\boldsymbol{\lambda}_{A}\right)$ & $0.08637873+0.6505825 i$ & $-0.05575262+1.282163 i$ \\
$\mathcal{R}^{(1)}\left(\boldsymbol{\lambda}_{B}\right)$ & $4.812087+0.8811173 i$ & $-5.332701-6.518506 i$ \\
$\mathcal{R}^{(1)}\left(\boldsymbol{\lambda}_{C}\right)$ & $0.05297897-4.432186 i$ & $-2.497722-22.42864 i$ \\
$\mathcal{R}^{(2)}\left(\boldsymbol{\lambda}_{A}\right)$ & $-2.385158+18.22971 i$ & $-28.12588+26.67761 i$ \\
$\mathcal{R}_{\mathrm{LC}}^{(2)}\left(\lambda_{A}\right)$ & $0.4123777+22.64313 i$ & $-1.450073+7.396238 i$ \\
$\mathcal{R}^{(2)}\left(\lambda_{B}\right)$ & $115.9528+18.71704 i$ & $17.16557-102.3377 i$ \\
$\mathcal{R}_{\mathrm{LC}}^{(2)}\left(\boldsymbol{\lambda}_{B}\right)$ & $144.2892-3.600533 i$ & $33.14649-134.9655 i$ \\
$\mathcal{R}^{(2)}\left(\boldsymbol{\lambda}_{C}\right)$ & $-36.87656-153.3540 i$ & $-26.92189-508.2138 i$ \\
$\mathcal{R}_{\mathrm{LC}}^{(2)}\left(\boldsymbol{\lambda}_{C}\right)$ & $-55.57522-190.2039 i$ & $76.13565-214.1456 i$ \\
\hline \hline
\end{tabular}

In the following, we consider the finite remainders

$$
\begin{aligned}
& \mathcal{R}^{(l)}(\lambda)=\mathcal{R}^{E,(l)}(\lambda)+\bar{\epsilon}_{5} \mathcal{R}^{O,(l)}(\lambda) \quad(l=1,2), \\
& \mathcal{R}_{\mathrm{LC}}^{(2)}(\lambda)=\tilde{c}_{1}\left[\mathcal{R}_{1}^{E,(2)}(\lambda)+\bar{\epsilon}_{5} \mathcal{R}_{1}^{O,(2)}(\lambda)\right] \\
& \mathcal{R}_{\mathrm{PL}}^{(2)}(\lambda)=\sum_{i=1,4,5,6,11} \tilde{c}_{i}\left[\mathcal{R}_{i}^{E,(2)}(\lambda)+\bar{\epsilon}_{5} \mathcal{R}_{i}^{O,(2)}(\lambda)\right],
\end{aligned}
$$

which include all color structures at one and two loop, just the leading-color two-loop contribution, or a combination of planar two-loop contributions, respectively. In Table I we present benchmark results for $\mathcal{R}^{(1)}, \mathcal{R}^{(2)}$, and $\mathcal{R}_{\mathrm{LC}}^{(2)}$ in the three independent helicity configurations, where we choose $q=u$ (up quark), $n_{f}=5$, the point

$$
\begin{aligned}
& s_{12}=157, \quad s_{23}=-43, \quad s_{34}=83, \\
& s_{45}=61, \quad s_{15}=-37, \quad \mu^{2}=100,
\end{aligned}
$$

and use PENTAGONMI [39] for the numerical evaluation of the pentagon functions. Interestingly, for this phase-space point, the leading-color finite remainder does not approximate well the full result. To investigate this further, in Fig. 1 we focus on the $u \bar{u}$ channel and plot the helicity-summed interference of the tree amplitude $\mathcal{A}^{(0)}$ with the two-loop finite remainders $\mathcal{R}_{X}^{(2)}=\mathcal{R}^{(2)}, \mathcal{R}_{\mathrm{LC}}^{(2)}$, and $\mathcal{R}_{\mathrm{PL}}^{(2)}$ as a function of the gluon transverse momentum. We fix the scattering energy as $\sqrt{s_{12}}=1 \mathrm{TeV}$, the invariant mass of the diphoton system as $\sqrt{s_{45}}=100 \mathrm{GeV}$, the transverse momentum and rapidity of the softer photon as $50 \mathrm{GeV}$ and -0.2 , respectively, and $\mu=\sqrt{s_{45}}$. Although further investigation is required, this analysis points to the importance of including virtual contributions beyond leading color. 


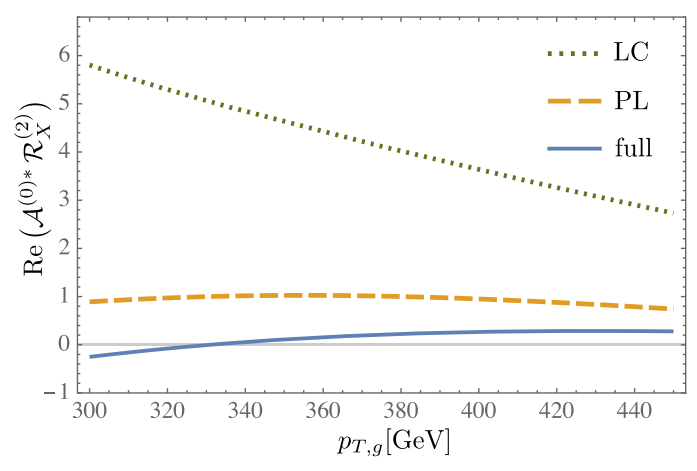

FIG. 1. Helicity-summed interference of tree-level amplitude with two-loop finite remainders for leading-color (dotted), leading-color plus planar-fermionic (dashed), and full-color contributions (solid) for the process $u \bar{u} \rightarrow g \gamma \gamma$ in dependence of the gluon transverse momentum.

To conclude, we have presented the first calculation of the complete, full-color two-loop QCD corrections to the helicity amplitudes of a $2 \rightarrow 3$ scattering process. Our calculation relied on recently developed techniques to handle multiloop multileg amplitudes and to perform the required reductions to master integrals. Our results provide the last missing amplitudes to describe prompt diphoton production at $\mathrm{N}^{3} \mathrm{LO} \mathrm{QCD}$, and, more exclusively, pairs of photons recoiling against hard QCD radiation at NNLO accuracy, and will enable highly accurate phenomenological studies of diphoton processes at hadron colliders.

In Ref. [82], we provide our complete analytical results for the one- and two-loop finite remainders for all helicity configurations and color factors, separated into parity even and odd contributions.

We thank Rene Poncelet for facilitating the comparison of the leading-color part against the results in [56]. We further thank Fabrizio Caola, Tiziano Peraro, and Gavin Salam for useful comments on the manuscript. B. A. and A. v. M. are supported in part by the National Science Foundation through Grant No. 2013859. The research of F. B. is supported by the ERC Starting Grant No. 804394 HipQCD. L. T. is supported by the Royal Society through Grant No. URF/R1/191125.

agarwalb@msu.edu

†federico.buccioni@physics.ox.ac.uk

†vmante@msu.edu

\$lorenzo.tancredi@physics.ox.ac.uk

[1] F. Tkachov, A theorem on analytical calculability of four loop renormalization group functions, Phys. Lett. 100B, 65 (1981).

[2] K. Chetyrkin and F. Tkachov, Integration by parts: The algorithm to calculate beta functions in 4 loops, Nucl. Phys. B192, 159 (1981).
[3] A. Kotikov, Differential equations method: New technique for massive Feynman diagrams calculation, Phys. Lett. B 254, 158 (1991).

[4] Z. Bern, L. J. Dixon, and D. A. Kosower, Dimensionally regulated pentagon integrals, Nucl. Phys. B412, 751 (1994).

[5] E. Remiddi, Differential equations for Feynman graph amplitudes, Nuovo Cimento A 110, 1435 (1997) [arXiv: hep-th/9711188].

[6] T. Gehrmann and E. Remiddi, Differential equations for two loop four point functions, Nucl. Phys. B580, 485 (2000).

[7] C. G. Papadopoulos, Simplified differential equations approach for master integrals, J. High Energy Phys. 07 (2014) 088.

[8] L. J. Dixon, Calculating scattering amplitudes efficiently, arXiv:hep-ph/9601359.

[9] N. Arkani-Hamed, J. L. Bourjaily, F. Cachazo, and J. Trnka, Local integrals for planar scattering amplitudes, J. High Energy Phys. 06 (2012) 125.

[10] A. Kotikov, The property of maximal transcendentality in the $N=4$ supersymmetric Yang-Mills, arXiv:1005.5029.

[11] J. M. Henn, Multiloop Integrals in Dimensional Regularization Made Simple, Phys. Rev. Lett. 110, 251601 (2013).

[12] A. B. Goncharov, Geometry of configurations, polylogarithms, and motivic cohomology, Adv. Math. 114, 197 (1995).

[13] E. Remiddi and J. Vermaseren, Harmonic polylogarithms, Int. J. Mod. Phys. A 15, 725 (2000).

[14] A. B. Goncharov, Multiple polylogarithms and mixed Tate motives, arXiv:math/0103059.

[15] A. B. Goncharov, M. Spradlin, C. Vergu, and A. Volovich, Classical Polylogarithms for Amplitudes and Wilson Loops, Phys. Rev. Lett. 105, 151605 (2010).

[16] F. Brown, The massless higher-loop two-point function, Commun. Math. Phys. 287, 925 (2009).

[17] J. Ablinger, J. Blümlein, and C. Schneider, Analytic and algorithmic aspects of generalized harmonic sums and polylogarithms, J. Math. Phys. (N.Y.) 54, 082301 (2013).

[18] E. Panzer, Algorithms for the symbolic integration of hyperlogarithms with applications to Feynman integrals, Comput. Phys. Commun. 188, 148 (2015).

[19] C. Duhr, H. Gangl, and J. R. Rhodes, From polygons and symbols to polylogarithmic functions, J. High Energy Phys. 10 (2012) 075.

[20] C. Duhr, Hopf algebras, coproducts and symbols: An application to Higgs boson amplitudes, J. High Energy Phys. 08 (2012) 043.

[21] C. Duhr and F. Dulat, PolyLogTools-Polylogs for the masses, J. High Energy Phys. 08 (2019) 135.

[22] J. Henn, B. Mistlberger, V. A. Smirnov, and P. Wasser, Constructing $d$-log integrands and computing master integrals for three-loop four-particle scattering, J. High Energy Phys. 04 (2020) 167.

[23] J. M. Henn and B. Mistlberger, Four-Gluon Scattering at Three Loops, Infrared Structure, and the Regge Limit, Phys. Rev. Lett. 117, 171601 (2016).

[24] J. M. Henn and B. Mistlberger, Four-graviton scattering to three loops in $\mathcal{N}=8$ supergravity, J. High Energy Phys. 05 (2019) 023. 
[25] F. Caola, A. von Manteuffel, and L. Tancredi, Di-Photon Amplitudes in Three-Loop Quantum Chromodynamics, Phys. Rev. Lett. 126, 112004 (2021).

[26] A. Hodges, Eliminating spurious poles from gauge-theoretic amplitudes, J. High Energy Phys. 05 (2013) 135.

[27] H. Ita, Two-loop Integrand decomposition into master integrals and surface terms, Phys. Rev. D 94, 116015 (2016).

[28] S. Badger, Automating QCD amplitudes with on-shell methods, J. Phys. Conf. Ser. 762, 012057 (2016).

[29] S. Abreu, F. Febres Cordero, H. Ita, M. Jaquier, B. Page, and M. Zeng, Two-Loop Four-Gluon Amplitudes from Numerical Unitarity, Phys. Rev. Lett. 119, 142001 (2017).

[30] H. A. Chawdhry, M. A. Lim, and A. Mitov, Two-loop fivepoint massless QCD amplitudes within the integration-byparts approach, Phys. Rev. D 99, 076011 (2019).

[31] S. Abreu, J. Dormans, F. Febres Cordero, H. Ita, M. Kraus, B. Page, E. Pascual, Ruf, and V. Sotnikov, Caravel: A $\mathrm{C}++$ framework for the computation of multi-loop amplitudes with numerical unitarity, Comput. Phys. Commun. 267, 108069 (2021).

[32] A. von Manteuffel and R. M. Schabinger, A novel approach to integration by parts reduction, Phys. Lett. B 744, 101 (2015).

[33] T. Peraro, Scattering amplitudes over finite fields and multivariate functional reconstruction, J. High Energy Phys. 12 (2016) 030.

[34] T. Peraro, FiniteFlow: Multivariate functional reconstruction using finite fields and dataflow graphs, J. High Energy Phys. 07 (2019) 031.

[35] X. Guan, X. Liu, and Y.-Q. Ma, Complete reduction of integrals in two-loop five-light-parton scattering amplitudes, Chin. Phys. C 44, 093106 (2020).

[36] C. G. Papadopoulos, D. Tommasini, and C. Wever, The pentabox master integrals with the simplified differential equations approach, J. High Energy Phys. 04 (2016) 078.

[37] T. Gehrmann, J. Henn, and N. Lo Presti, Pentagon functions for massless planar scattering amplitudes, J. High Energy Phys. 10 (2018) 103.

[38] D. Chicherin, T. Gehrmann, J. Henn, N. Lo Presti, V. Mitev, and P. Wasser, Analytic result for the nonplanar hexa-box integrals, J. High Energy Phys. 03 (2019) 042.

[39] D. Chicherin and V. Sotnikov, Pentagon functions for scattering of five massless particles, J. High Energy Phys. 12 (2020) 167.

[40] T. Gehrmann, J. M. Henn, and N. A. Lo Presti, Analytic form of the Two-Loop Planar Five-Gluon All-Plus-Helicity Amplitude in QCD, Phys. Rev. Lett. 116, 062001 (2016); Erratum, Phys. Rev. Lett.116, 189903(E) (2016).

[41] S. Badger, C. Broennum-Hansen, H. B. Hartanto, and T. Peraro, First Look at Two-Loop Five-Gluon Scattering in QCD, Phys. Rev. Lett. 120, 092001 (2018).

[42] S. Abreu, F. Febres Cordero, H. Ita, B. Page, and M. Zeng, Planar two-loop five-gluon amplitudes from numerical unitarity, Phys. Rev. D 97, 116014 (2018).

[43] S. Abreu, L. J. Dixon, E. Herrmann, B. Page, and M. Zeng, The Two-Loop Five-Point Amplitude in $\mathcal{N}=4$ superYang-Mills Theory, Phys. Rev. Lett. 122, 121603 (2019).

[44] S. Abreu, J. Dormans, F. Febres Cordero, H. Ita, and B. Page, Analytic Form of Planar Two-Loop Five-Gluon
Scattering Amplitudes in QCD, Phys. Rev. Lett. 122, 082002 (2019).

[45] S. Abreu, F. Febres Cordero, H. Ita, B. Page, and V. Sotnikov, Planar two-loop five-parton amplitudes from numerical unitarity, J. High Energy Phys. 11 (2018) 116.

[46] S. Abreu, L. J. Dixon, E. Herrmann, B. Page, and M. Zeng, The two-loop five-point amplitude in $\mathcal{N}=8$ supergravity, J. High Energy Phys. 03 (2019) 123.

[47] S. Abreu, B. Page, E. Pascual, and V. Sotnikov, Leadingcolor two-loop QCD corrections for three-photon production at hadron colliders, J. High Energy Phys. 01 (2021) 078 .

[48] S. Badger, C. Brønnum-Hansen, H. B. Hartanto, and T. Peraro, Analytic helicity amplitudes for two-loop five-gluon scattering: The single-minus case, J. High Energy Phys. 01 (2019) 186.

[49] D. Chicherin, T. Gehrmann, J. M. Henn, P. Wasser, Y. Zhang, and S. Zoia, Analytic Result for a Two-Loop FiveParticle Amplitude, Phys. Rev. Lett. 122, 121602 (2019).

[50] D. Chicherin, T. Gehrmann, J. M. Henn, P. Wasser, Y. Zhang, and S. Zoia, The two-loop five-particle amplitude in $\mathcal{N}=8$ supergravity, J. High Energy Phys. 03 (2019) 115.

[51] H. A. Chawdhry, M. Czakon, A. Mitov, and R. Poncelet, Two-loop leading-color helicity amplitudes for three-photon production at the LHC, J. High Energy Phys. 06 (2021) 150.

[52] G. De Laurentis and D. Maître, Two-loop five-parton leading-colour finite remainders in the spinor-helicity formalism, J. High Energy Phys. 02 (2021) 016.

[53] B. Agarwal, F. Buccioni, A. von Manteuffel, and L. Tancredi, Two-loop leading colour QCD corrections to $q \bar{q} \rightarrow \gamma \gamma g$ and $q g \rightarrow \gamma \gamma q$, J. High Energy Phys. 04 (2021) 201.

[54] S. Badger, H. B. Hartanto, and S. Zoia, Two-Loop QCD Corrections to $W b \bar{b}$ Production at Hadron Colliders, Phys. Rev. Lett. 127, 012001 (2021).

[55] S. Abreu, F. F. Cordero, H. Ita, B. Page, and V. Sotnikov, Leading-color two-loop QCD corrections for three-jet production at hadron colliders, J. High Energy Phys. 07 (2021) 095.

[56] H. A. Chawdhry, M. Czakon, A. Mitov, and R. Poncelet, Two-loop leading-colour QCD helicity amplitudes for twophoton plus jet production at the LHC, J. High Energy Phys. 07 (2021) 164.

[57] H. A. Chawdhry, M. L. Czakon, A. Mitov, and R. Poncelet, NNLO QCD corrections to three-photon production at the LHC, J. High Energy Phys. 02 (2020) 057.

[58] S. Kallweit, V. Sotnikov, and M. Wiesemann, Triphoton production at hadron colliders in NNLO QCD, Phys. Lett. B 812, 136013 (2021).

[59] S. Badger, D. Chicherin, T. Gehrmann, G. Heinrich, J. Henn, T. Peraro, P. Wasser, Y. Zhang, and S. Zoia, Analytic Form of the Full Two-Loop Five-Gluon All-Plus Helicity Amplitude, Phys. Rev. Lett. 123, 071601 (2019).

[60] N. Byers and C. Yang, Physical regions in invariant variables for $\mathrm{n}$ particles and the phase-space volume element, Rev. Mod. Phys. 36, 595 (1964).

[61] T. Peraro and L. Tancredi, Physical projectors for multi-leg helicity amplitudes, J. High Energy Phys. 07 (2019) 114.

[62] T. Peraro and L. Tancredi, Tensor decomposition for bosonic and fermionic scattering amplitudes, Phys. Rev. D 103, 054042 (2021). 
[63] V. Del Duca, F. Maltoni, Z. Nagy, and Z. Trocsanyi, QCD radiative corrections to prompt diphoton production in association with a jet at hadron colliders, J. High Energy Phys. 04 (2003) 059.

[64] P. Nogueira, Automatic Feynman graph generation, J. Comput. Phys. 105, 279 (1993).

[65] J. Vermaseren, New features of FORM, arXiv:math-ph/0010025.

[66] See Supplemental Material at http://link.aps.org/supplemental/ 10.1103/PhysRevLett.127.262001 for the definition of the integral families, details on the renormalization and infrared subtraction procedures and the discussion on the computation of the set of non-independent helicity amplitudes.

[67] S. Laporta, High precision calculation of multiloop Feynman integrals by difference equations, Int. J. Mod. Phys. A 15, 5087 (2000).

[68] D. Bendle, J. Boehm, M. Heymann, R. Ma, M. Rahn, L. Ristau, M. Wittmann, Z. Wu, and Y. Zhang, Two-loop fivepoint integration-by-parts relations in a usable form, arXiv:2104.06866.

[69] A. von Manteuffel and C. Studerus, Reduze 2-Distributed Feynman integral reduction, arXiv:1201.4330.

[70] P. Wang, M. Guy, and J. Davenport, $P$-adic reconstruction of rational numbers, SIGSAM Bulletin 16, 2 (1982).

[71] J. Gluza, K. Kajda, and D. A. Kosower, Towards a basis for planar two-loop integrals, Phys. Rev. D 83, 045012 (2011).

[72] K. J. Larsen and Y. Zhang, Integration-by-parts reductions from unitarity cuts and algebraic geometry, Phys. Rev. D 93, 041701(R) (2016).

[73] J. Böhm, A. Georgoudis, K. J. Larsen, M. Schulze, and Y. Zhang, Complete sets of logarithmic vector fields for integration-by-parts identities of Feynman integrals, Phys. Rev. D 98, 025023 (2018).

[74] B. Agarwal, S. P. Jones, and A. von Manteuffel, Two-loop helicity amplitudes for $g g \rightarrow Z Z$ with full top-quark mass effects, J. High Energy Phys. 05 (2021) 256.

[75] M. Heller and A. von Manteuffel, MultivariateApart: Generalized partial fractions, Comput. Phys. Commun. 271, 108174 (2022).

[76] R. Lewis, Computer Algebra System Fermat, http://www. bway.net/ lewis.

[77] S. Abreu, J. Dormans, F. Febres Cordero, H. Ita, B. Page, and V. Sotnikov, Analytic form of the planar two-loop fiveparton scattering amplitudes in QCD, J. High Energy Phys. 05 (2019) 084.

[78] J. Boehm, M. Wittmann, Z. Wu, Y. Xu, and Y. Zhang, IBP reduction coefficients made simple, J. High Energy Phys. 12 (2020) 054.

[79] W. Decker, G.-M. Greuel, G. Pfister, and H. Schönemann, SINGULAR 4-2-0-A computer algebra system for polynomial computations, http://www.singular.uni-kl.de (2020).

[80] S. Catani, The Singular behavior of QCD amplitudes at two loop order, Phys. Lett. B 427, 161 (1998).

[81] F. Buccioni, J.-N. Lang, J. M. Lindert, P. Maierhöfer, S. Pozzorini, H. Zhang, and M. F. Zoller, OpenLoops 2, Eur. Phys. J. C 79, 866 (2019).

[82] B. Agarwal, F. Buccioni, A. von Manteuffel and L. Tancredi, Github repository, Version: 1.0 (2021), https:// gitlab.msu.edu/vmante/aajamp-symb. 